Romero-Rodrigo, M. \& López-Marí, M. (2021). Luces, sombras y retos del profesorado entorno a la gamificación apoyada en TIC: un estudio con maestros en formación. Revista Electrónica Interuniversitaria de Formación del Profesorado, 24(2), 167-179.

\title{
Luces, sombras y retos del profesorado entorno a la gamificación apoyada en TIC: un estudio con maestros en formación
}

\author{
Mercedes Romero Rodrigo(1), María López Marí( ${ }^{(2)}$ \\ Universidad Camilo José Cela ${ }^{(1)}$, Madre Vedruna Sagrado Corazón FEC ${ }^{(2)}$
}

\section{Resumen}

El presente trabajo tiene como propósito identificar y describir las percepciones de un grupo de estudiantes de Máster en cuanto a los efectos positivos y negativos de la introducción de estrategias de gamificación apoyadas en el uso de TIC en sus alumnos, así como los retos a los que se enfrenta el profesorado en este sentido. El diseño de esta investigación se corresponde a un estudio de caso no experimental de tipo interpretativo, donde la recogida de información se planteó entorno al desarrollo de una actividad académica de tipo reflexivo-argumentativo teniendo en cuenta el contexto propio de los estudiantes junto con su experiencia profesional. Como principales ventajas se aludieron a los beneficios relacionados con la motivación, actitudes y emociones ante el aprendizaje, seguidos de mejoras en aspectos sociales y comportamentales. Por el contrario, las dudas generadas en relación a aspectos conceptuales y de contenido sumado a la sobreexposición de la tecnología, son las preocupaciones más habituales. En cuanto a los retos se subrayó la importancia de hacerles frente desde una triple vertiente que integrase tanto una perspectiva pedagógica, como digital y económica. Desde esta premisa urgen a revisar desde la concepción y formulación de propuestas formativas integrales destinadas a los profesores, las necesidades de equipamiento en los centros, hasta la revisión de estrategias didácticas gamificadas a fin de tener en cuenta la diversidad existente en las aulas.

\section{Palabras clave}

Gamificación; TIC; Innovación educativa; Formación del profesorado.

\section{Contacto:}

Mercedes Romero Rodrigo, mmromero@ucjc.edu , Universidad Camilo José Cela. Facultad de Educación. C/ Castillo de Alarcón, 49. Villafranca del Castillo, 28692 Madrid. 


\title{
Lights, shadows and challenges of teachers around gamification supported by ICT: a study with teachers in training
}

\begin{abstract}
The purpose of this paper is to identify and describe the perceptions of a group of Master's students regarding the positive and negative effects of the introduction of gamification strategies supported by the use of ICT in their students, as well as the challenges facing teachers in this regard. The design of this research corresponds to an interpretative nonexperimental case study, where the collection of information was based on the development of an academic activity of a reflexive-argumentative type taking into account the students' own context as well as their professional experience. The main advantages were the benefits related to motivation, attitudes and emotions towards learning, followed by improvements in social and behavioral aspects. On the contrary, the doubts generated in relation to conceptual aspects and content added to the overexposure of technology, are the most common concerns. Regarding the challenges, the importance of addressing them from a threefold perspective that integrates both a pedagogical, digital and economic perspective was stressed. They need to review from the conception and formulation of comprehensive training proposals for teachers, the equipment needs in schools, until the review of gamified teaching strategies to take account of the diversity in the classroom.
\end{abstract}

\section{Key words}

Gamification; TIC; Educational innovation; Teacher training

\section{Introducción}

La transformación digital del sector educativo ha suscitado nuevos modos de interacción entre las personas y el conocimiento, al que se puede acceder de forma inmediata, a través de múltiples dispositivos y fuentes de información sin barreras espacio-temporales. Este hecho, tal y como apuntan Marín et al. (2019), ha ocasionado que los profesionales de la educación desempeñen un rol muy importante, ya que no solo deben conocer la materia que imparten, sino que también deben diseñar y gestionar la información que posteriormente transmitirán al alumnado.

Por lo tanto, aunque la incorporación de la tecnología educativa implica múltiples variables, organizativas, legislativas o económicas, la concepción del profesorado, el grado de formación y dominio que tengan de estos recursos será un elemento esencial (Cabero \& Marín, 2014). Debido a que no se trata tanto de que el profesorado conozca y maneje competentemente herramientas digitales, sino de dotarlos de habilidades para la práctica, siendo capaces de analizar, diseñar e integrar las tecnologías de forma adecuada en sus programaciones curriculares (Ballesta, 1993). Concepción que coincide con la definición elaborada por el Instituto Nacional de Tecnologías Educativas y de Formación del Profesorado (INTEF, 2017), que define la competencia digital como "el uso creativo, crítico y seguro de las tecnologías de información y comunicación para alcanzar los objetivos relacionados con el trabajo, la empleabilidad, el aprendizaje, el tiempo libre, la inclusión y la participación en la sociedad" (p. 9). 
Este escenario que se plantea, exigirá al profesorado emplear nuevas habilidades y destrezas que consigan repercutir positivamente en el proceso de enseñanza y aprendizaje (Fuentes \& González, 2017). Siendo necesario, como señala Peirats et al. (2019), que el profesorado tenga el liderazgo en la digitalización de la educación, pues como se ha comprobado a lo largo de los años, en cualquier reforma e innovación educativa la figura del profesorado es clave.

Un ejemplo reciente de ello es la situación generada por la COVID-19, en la que el profesorado se vio obligado a buscar nuevas estrategias y herramientas digitales que permitieran adaptar el proceso de enseñanza y garantizar la continuidad del curso (RoigVila et al., 2021). Aunque, como recoge Cabero y Valencia (2021) en su investigación, no siempre fueran lo suficientemente habilidosos en la creación de entornos formativos enriquecidos por la tecnología. A causa de que todavía prevalece un modo transmisivo de la información en el proceso de enseñanza y aprendizaje y existe una falta de experiencias previas acordes con las nuevas formas de aprender del siglo XXI (Álvarez \& Kilbourn, 2005).

Una estrategia didáctica que se está implementando en la actualidad con resultados positivos, es la gamificación apoyada en el uso de TIC (Silva et al., 2020) y que se caracteriza por unificar el uso de las tecnologías educativas, mediante aplicaciones como Classcraft, Classdojo o Kahoot!, con un aprendizaje más activo, cooperativo e interactivo. Concretamente, este término proviene del vocablo inglés game, que significa juego, y se define como el uso de los elementos del juego que motivan a las personas en otros ámbitos no lúdicos, como pueda ser un aula (McGonigal, 2011). Con el objetivo de que, como señalan Rodríguez y Santiago (2015), las personas realicen algo que no siempre les apetece, interesa o motiva usando el juego.

En sus orígenes, la gamificación se empezó a utilizar en el mundo empresarial (Cortizo et al., 2011), aunque pronto trascendió a otros ámbitos como el de la salud, el medio ambiente o el educativo. En la expansión de la gamificación se considera que tuvo mucho que ver el crecimiento de la industria del videojuego, que lo convirtió en un componente fundamental de la cultura popular moderna (Maté, 2021). Y que ha dado lugar a lo que hoy en día se conoce como la generación $G$, que como ocurrió con sus predecesoras, la generación $Y$, marcada por la expansión de Internet, o la generación Z, influenciada por el inicio de la digitalización, se caracteriza por estar conformada por personas acostumbradas a los medios digitales y a las redes sociales, pero sobre todo por ser consumidoras de juegos electrónicos (Hernández et al., 2014; Prensky, 2001).

Entre las potencialidades de la gamificación se subraya, como apunta Vázquez-Ramos (2021), la utilización de estrategias psicológicas propias de los videojuegos que generan ese engagement de los usuarios. Fundamentadas, sobre todo, en el reconocimiento, el logro, la competencia, la colaboración o la autoexpresión (Sánchez, 2015). Gracias a las cuales, aquel alumnado que, por lo general, se muestra menos motivado en un proceso de enseñanzaaprendizaje tradicional esté más implicado y se muestre más participativo (Salvador-García, 2021).

Del mismo modo, se destaca que esta estrategia también favorece la creación de entornos educativos más atractivos e inclusivos (Lee \& Hammer, 2011); fomenta la retroalimentación por parte del profesorado, ayudando al alumnado a progresar y continuar con el siguiente nivel (Kapp, 2012); o finalmente, como subraya Putz et al. (2020), genera la adquisición de conocimientos independientemente de la edad o el género. Ya que, como se ha podido comprobar en este trabajo, las experiencias de aprendizaje gamificado están presentes en las diferentes etapas educativas obligatorias, tanto en Educación Primaria (Cruz \& Otero, 2017; García-Vandewalle, 2017; Garmen et al., 2019; Gil, 2019; Ferrer et al., 2018; Prieto, 2018; 
Subinas \& Berciano, 2019; Villena et al., 2018), como en Educación Secundaria y Bachillerato (Hernández, 2018; Obando-Bastidas et al., 2018; Quintero et al., 2018; Vergara et al., 2019).

Atendiendo a todo lo expuesto, este trabajo se va a centrar en el análisis de la gamificación como estrategia didáctica y en la profundización de su conocimiento. Para ello, se pretende identificar y describir las percepciones de un grupo de estudiantes de Máster, maestros y maestras de Educación Infantil, Primaria y Secundaria, sobre la introducción de estrategias de gamificación apoyadas en las TIC. Con el objetivo de discernir, desde sus puntos de vista, los efectos positivos y negativos que encuentran así como los retos futuros a los que se enfrentan.

\section{Método}

Este trabajo se enmarca dentro de una experiencia de innovación educativa en el Máster Universitario en Tecnología Digital Aplicada a la Práctica Docente, concretamente en la asignatura Proyectos de innovación y últimas tendencias digitales del curso académico 2019-2020 de la Universidad Camilo José Cela. Como se expuso con anterioridad, el propósito de la investigación tuvo por objeto identificar y describir las percepciones de los estudiantes en torno a la gamificación apoyada en el uso de la tecnología como estrategia didáctica, descifrando aspectos positivos, negativos así como retos a hacer frente por parte de los maestros y maestras.

En cuanto a la muestra inicial del estudio indicar que estaba formada por 196 estudiantes y que se corresponden al conjunto de matriculados en la materia. Finalmente la muestra resultó ser de 183, ya que 13 de ellos no participaron en la actividad diseñada y que permitió la recogida de datos, tal y como se detalla más adelante. De acuerdo con dicha muestra revelar que un $22.95 \%$ estaba constituida por hombres mientras que $77.05 \%$ fueron mujeres.

Siguiendo con el proceso de recogida de información, la principal fuente de obtención de la misma fue una actividad que formaba parte de la evaluación continua de la asignatura. En ella el estudiantado debía reflexionar desde el punto de vista de los alumnos de etapas educativas obligatorias, sobre las ventajas e inconvenientes que suponía la incorporación de la gamificación apoyada en el uso de las TIC en los procesos de enseñanza y aprendizaje. A su vez y a partir de dicha descripción, cabía profundizar sobre los retos a los que se enfrentaban como profesores en este sentido. Para el análisis de los datos obtenidos referidos a los aspectos positivos y negativos se empleó una matriz de contenido creada a propósito de esta investigación y cuyas dimensiones de análisis partieron de la definición de competencia (OCDE, 2003), a saber: 1. Habilidades prácticas; 2. Conocimientos; 3. Motivación; 4. Aspectos éticos; 5. Actitudes; 6 . Emociones; 7. Sociales y comportamentales. Se tomaron en consideración dichas dimensiones de análisis con la finalidad de lograr una mirada integral de los aprendizajes trascendiendo de este modo de aspectos puramente curriculares. En cuanto a las dimensiones de análisis referidas a los retos se tomó como punto de partida el trabajo de Gairín y Castro (2016) sobre los obstáculos identificados por los docentes para un mejor aprovechamiento de las TIC en los procesos educativos, agrupándolas en: 1 . Retos a nivel pedagógico; 2 . Retos a nivel tecnológico; 3. Retos a nivel social o de contexto.

En una primera fase se procedió a realizar un vaciado de datos a partir de las respuestas dadas por los alumnos, categorizándolas atendiendo a las dimensiones enunciadas. Después, en la fase siguiente, fue acometida la revisión e interpretación de los datos teniendo en cuenta los objetivos fijados (ventajas, inconvenientes y retos). 


\section{Resultados}

Seguidamente pasamos a abordar los hallazgos confrontados con respecto al tema que nos ocupa, la gamificación mediada por las TIC como estrategia didáctica: luces, sombras y retos aducidos por maestros y maestras en formación. De una parte se expondrán los resultados referidos a las dimensiones destacadas en cada efecto (positivo y negativo) para, a continuación, profundizar en los retos aludidos por los participantes en la investigación.

\section{Efectos positivos}

En relación a las ventajas enumeradas por los estudiantes, sobresalieron tres dimensiones de manera notable: 3 . Motivación, 5. Actitudes y 6. Emociones; prácticamente tres cuartas partes de los participantes centraron su reflexión en torno a ellas. Por consiguiente la reciprocidad que establecieron entre motivación, actitud y emociones fue estrecha, afectando unas sobre las otras.

Tabla 1.

Número de argumentos positivos expuestos

\begin{tabular}{ll}
\hline Dimensiones & N \\
\hline 1. Habilidades prácticas & 12 \\
\hline 2. Conocimientos & 23 \\
\hline 3. Motivación & 74 \\
\hline 4. Aspectos éticos & 16 \\
\hline 5. Actitudes & 60 \\
\hline 6. Emociones & 49 \\
\hline 7. Sociales y comportamentales & 36 \\
\hline
\end{tabular}

Nota: 68 estudiantes realizaron aportaciones en dos o más dimensiones.

El argumento más habitual ponía de manifiesto que la gamificación apoyada en el uso de TIC producía un incremento en la motivación, actitudes positivas e interés por el aprendizaje. La predisposición hacia el estudio, sumado al feedback instantáneo así como a un incremento de la motivación de tipo intrínseco, fueron otros de los argumentos más repetidos. Sobre esto último, los participantes de la investigación establecieron relaciones interesantes con el incremento de la autonomía por parte del alumnado, subrayando que en la medida que los alumnos y alumnas van superando retos y logrando recompensas, insignias y demás, se genera una inercia que actúa como motor de la iniciativa tanto individual como grupal. La superación de retos, las evidencias en barras de progreso y reconocimiento de logros parece contribuir a que los alumnos deseen hacer más cosas por ellos mismos, produciendo sentimientos positivos no únicamente hacia las situaciones de aprendizaje sino también, por ejemplo, mejorando la autoestima y el autoconcepto. La posibilidad de aprender ante el error, el ambiente relajado y la seguridad emocional son otros de los aspectos mejor valorados, ya que afirman que contribuyen a generar un clima tranquilo, favoreciendo el florecimiento de emociones positivas como la curiosidad, la diversión, el interés, la satisfacción y el orgullo ante los logros: 
La gamificación permite por tanto trabajar en la resiliencia y la aceptación del fallo como algo normal, en el proceso de aprendizaje, lo cual facilita el aprendizaje futuro de otras materias. Por otro lado, la creación de emociones positivas provoca un vínculo mayor con el alumno, que le dirigen hacia la realización de una tarea o acción determinada, al tiempo que generan un mayor compromiso por su parte (E25, línea 29-33).

Otra ventaja de las TICs y la gamificación es que fomenta la cooperación. Lo que permitirá a los alumnos a llegar a ciertas metas, pidiendo en ocasiones ayuda y en otras siendo los que la dispongan. Es decir ellos ayudan a solventar los problemas sin necesidad que sea el profesor el que los resuelva, como sucede en modelos más tradicionales. El alumno por tanto toma un papel más relevante y proactivo (E151, línea 18-22).

A tenor de las siguientes citas ilustrativas resaltar la relación con la última de las dimensiones, aspectos sociales y comportamentales. A este respecto, y teniendo en cuenta que la mayoría de las aportaciones se centran en valorar las ventajas a nivel individual, destacar que cuanto los estudiantes hacen referencia a las implicaciones positivas a nivel de grupo suelen reseñar la colaboración y cooperación para el logro de objetivos comunes. Es decir, entienden que apostar por la gamificación, siempre y cuando se plantee en un entorno donde prime el trabajo en equipo, contribuirá a desarrollar satisfactoriamente competencias sociales y comportamentales. Tal y como se observará en el siguiente apartado, este punto es un tema que suele generar mayor controversia y crítica por los participantes de la investigación, ya que han observado que una propuesta no basada en este principio podría ser conducente a un excesivo individualismo y rivalidad entre el alumnado.

Finalizando mencionar que las valoraciones que atañen al resto de dimensiones (1. Habilidades prácticas; 2 . Conocimientos; 4 . Aspectos éticos;) han sido mínimas y cuando se ha hecho referencia a ellas ha sido común referirse por extensión a la mejora de las competencias digitales, alfabetización digital y/o uso responsable de las TIC.

\section{Efectos negativos}

Así como cuando se analizaron los resultados atribuidos a los efectos positivos se evidenció que existía una clara inclinación en las dimensiones referidas a la motivación, actitudes y emociones, en el caso de los efectos negativos se ha podido constatar que principalmente las reflexiones se centraron en aspectos conceptuales (conocimientos), actitudinales, sociales y comportamentales (dimensiones 2, 5 y 7 ), siendo escasas las referidas al resto de dimensiones.

Tabla 2.

Número de argumentos negativos expuestos

\begin{tabular}{ll}
\hline Dimensiones & N \\
\hline 1. Habilidades prácticas & 6 \\
\hline 2. Conocimientos & 61 \\
\hline 3. Motivación & 18 \\
\hline 4. Aspectos éticos & 4 \\
\hline
\end{tabular}




\begin{tabular}{ll}
\hline Dimensiones & $\mathbf{N}$ \\
\hline 5. Actitudes & 48 \\
\hline 6. Emociones & 15 \\
\hline 7. Sociales y comportamentales & 69 \\
\hline
\end{tabular}

Nota: 46 estudiantes realizaron aportaciones en dos o más dimensiones.

Ciertamente se constata una preocupación real en cuanto a la posibilidad de aprendizajes incompletos o superficiales como consecuencia de un planteamiento didáctico liviano, donde la gamificación sea entendida más como el juego por el juego que como estrategia didáctica. Con relación a esta cuestión los estudiantes señalaron que una atención excesiva por el soporte o herramienta digital, descuidando el sentido didáctico de la propuesta, era un error de planteamiento que cabía evitar:

Entre las desventajas: encontramos que el acceso a ellas fuera del aula es inviable en algunos casos porque los alumnos no tienen medios en casa para continuar las tareas, las distracciones que esta metodología pueda ocasionar centrándose más en la herramienta digital que en los contenidos (...) (E35, línea 27-31).

(...) es que tanto juego, puede suponer que los alumnos no se tomen en serio el aprendizaje y se centren más en jugar suponiendo más una distracción que una herramienta de aprendizaje e incluso provocar el aislamiento de los alumnos. También se debe tener en cuenta que las dinámicas de juego no desarrollen excesiva competitividad (E164, línea 5256).

Por otro lado y como adelantamos en el punto anterior, una de las principales preocupaciones estuvo ligada a cuestiones actitudinales, de las que destacan las siguientes por su reiteración: problemas de distanciamiento social; competencia y rivalidad insana en el logro de los objetivos, desembocando en actitudes competitivas y falta de cooperación dentro del grupo (lo importante no es lograr la insignia o recompensa, sino llegar en primer lugar); dispersión, poca atención y motivación ante actividades no gamificadas; abuso y sobreexposición a las TIC. Sobre este último aporte se evidencia la inquietud sobre el tiempo que los alumnos pasan delante de las pantallas y la vacilación sobre la relación con el desarrollo de conductas adictivas (tecnoadicción) como consecuencia del abuso de las TIC.

(...) también existen aspectos negativos de la incorporación de las TIC, uno de los más preocupantes es la adicción que provocan. Cada vez son más los niños pequeños que utilizan dispositivos digitales para entretenerse, ver videos, jugar a juegos, así como cada vez son más los jóvenes que crean sus propios perfiles en redes sociales a edades tempranas (E103, línea 21-25).

Conforme a lo expuesto y siendo complicado circunscribir por su carácter transversal a una dimensión concreta, se enuncian a continuación dos preocupaciones presentes en las reflexiones que conviene no pasar por alto dado su alcance: efectos nocivos en la salud (cansancio visual y problemas posturales como los más comunes, a los que cabría sumar las 
conductas abusivas antes reseñadas) y consecuencias derivadas de la brecha digital. Si bien el primero de ellos podría incluirse dentro de las consecuencias nocivas de las dimensiones 5 y 7, en el caso de la brecha digital cabe detenerse un poco más: no disponer del equipamiento óptimo amén de una conectividad adecuada, puede terminar por acarrear la exclusión de estos estudiantes. $Y$ es que la brecha digital, con sus diferentes aristas, también es uno de los retos futuros a los que hacer frente tal y como queda reflejado en el siguiente apartado.

\section{Retos futuros para el profesorado}

Tres son los mantras que han venido repitiéndose en prácticamente la totalidad de las reflexiones de los estudiantes, en la mayoría de los casos uno de ellos ha estado presente y en cerca de la mitad de las participaciones se ha dado al menos otro aunque fuera de manera superficial:

- Ausencia de formación tecnológica y pedagógica sobre el tema en cuestión y que los profesores han ido salvando gracias a su carácter autodidacta. En este sentido, la apuesta no solo de la mejora de las competencias digitales, sino de formación práctica que permita la inclusión digital pedagógica, es señalada como fundamental.

- Retos ligados a la personalización de los aprendizajes de manera que la gamificación, como estrategia didáctica, permita también ajustar los objetivos de aprendizaje (construcción de itinerarios alternativos para el alcance de logros y metas): evitar tender a la homogenización del grupo, descuidando la atención a la diversidad.

- Compromiso por parte de los poderes públicos para minimizar la brecha digital existente en los centros con pocos recursos, lo que imposibilita introducir estrategias didácticas gamificadas apoyadas en un uso de las TIC de manera integral. Es decir, ir más allá de aplicaciones que se centren en la creación de avatares y la gratificación en forma puntos o recompensas.

Estos resultados muestran que los estudiantes en formación divisan a futuro que los retos a los que hacer frente tienen una triple vertiente estrechamente relacionadas: pedagógica, digital y económica. Acometer estos retos por separado, dados los resultados obtenidos, parece ser una decisión descartada vistas las relaciones establecidas entre ellas durante el análisis. Y es que la interdependencia de éstas hace que la propuesta de actuación pueda resultar insuficiente sino es abordada de manera exhaustiva.

\section{Discusión y conclusiones}

No es de extrañar que el creciente interés sobre la gamificación apoyada en el uso de TIC se produzca a la par del desarrollo tecnológico, favorecido en gran medida por la digitalización de nuestra sociedad así como por la accesibilidad de la tecnología en términos de reducción de costes (precio) de dispositivos (Gabarda et al., 2020). Sin ir más lejos, la producción científica sobre esta cuestión se ha incrementado progresivamente en los últimos años, coincidiendo con la predicción realizada en el informe Horizon en 2014 y en el que se vaticinó que nos encontrábamos ante un estrategia didáctica emergente que eclosionaría con fuerza en años posteriores (Johnson et al., 2014). Basta con acudir a las principales bases de datos para verificar la cantidad de publicaciones relacionadas con este tema, ya sean trabajos en torno a experiencias reales en disciplinas y etapas diversas o estudios de tipo bibliográfico, como por ejemplo en Trabajos Fin de Grado, Máster y Tesis Doctorales. 
A propósito de los resultados obtenidos subrayar las fortalezas percibidas por los participantes a la hora de aplicar la gamificación como estrategia. La mayoría de ellos destaca su potencial para motivar, generar emociones y favorecer una actitud más proactiva del alumnado (Ferrer et al., 2018; Peirats et al., 2019) gracias al poder que ejerce sobre ellos el uso de mecánicas propias del juego como los retos, puntos y recompensas (Dela Cruz \& Palaoag, 2019). Cabe señalar que se liga la eficacia de tales iniciativas al acompañamiento de un feedback inmediato y continuo, lo que conduce a mayor autonomía entre el alumnado y a la vez de a un mayor grado de compromiso con las actividades propuestas. Por último, entre los beneficios que puede reportar esta estrategia de las indicadas por los participantes en el estudio, destacan en positivo la capacidad para fomentar el trabajo cooperativo a través de agrupamientos heterogéneos. Esta organización, a su vez, pretende responder tanto a las necesidades individuales como a las capacidades diversas que puedan darse en un aula. Teniendo esto presente y sumado a un papel más activo en los procesos de aprendizaje, como apuntó Gil (2019), se contribuye a que los alumnos y alumnas migren desde una recepción pasiva de conocimientos a una actividad caracterizada por su protagonismo y tomando como base un aprendizaje colaborativo y social (Hernández, 2018; Prieto, 2018).

En relación a los aspectos más controvertidos incidir en la preocupación constatada sobre la poca profundización o ligereza a la hora de abordar ciertos contenidos cuando la propuesta se focaliza en exceso en el componente lúdico, descuidando la finalidad educativa de la propia acción. Dicha inquietud coincide con los aportes de investigaciones como las de Carpena et al. (2012) y Marín (2015), los cuales advirtieron que gamificar no debe limitarse a la ludificación de la enseñanza. En línea con lo expuesto, los participantes en esta investigación subrayaron que gamificar para divertir, motivar y/o hacer algo diferente (sin más reflexión sobre la propia praxis), ya sea por seguir una moda o incluir herramientas punteras (como pudieran ser Kahoot! o Quizizz), careciendo de un propósito educativo claro y conectado con el currículum, era un error (Vázquez-Ramos, 2021). En este sentido cabe reflexionar sobre el propio papel del profesorado en las dinámicas gamificadas, resultando primordial tomar un papel activo durante el desarrollo de las tareas: analizar y debatir con la clase, o por ejemplo, suscitar el aprendizaje a partir de los errores del grupo en su conjunto, contribuirá a construir un conocimiento sólido a partir de la experiencia vivida por los alumnos así como a su transferencia a otros contextos (Peirats, et al., 2019; Vergara et al. 2019). Sin lugar a dudas esta propuesta se erige como punto de partida para superar otro de los efectos perversos reseñados por los estudiantes: el efecto contraproducente de dinámicas gamificadas excesivamente individualistas. Y es que la vacilación sobre los efectos en el desarrollo particular de cada alumno, la pérdida de oportunidades de aprendizaje entre compañeros y la competencia insana entre ellos podría desencadenar en una merma de competencias de tipo social. Cabe, por tanto, diseñar estrategias gamificadas que fomenten el aprendizaje colectivo por encima del individual.

Resulta de interés detenerse en los retos señalados por los estudiantes con respecto a su futuro inmediato a la hora de diseñar actuaciones gamificadas. Si bien es cierto que existe unanimidad por parte de los participantes sobre la parquedad o ausencia de formación en este sentido, el carácter autodidacta de su aprendizaje y la necesidad de mayor compromiso por parte de la administración, no menos es cierto que estamos ante demandas que vienen siendo una constante entre el profesorado ya sea en este u otros temas (Gabarda et al., 2017; Gabarda et al., 2020; Peirats et al., 2015; Romero et al., 2014). Aún con todo no deja de ser llamativo que los desafíos apunten en esta dirección dada la naturaleza del Máster en curso y el carácter práctico de la asignatura en particular. Una de las explicaciones que podrían contribuir a comprender este fenómeno es el perfil de los participantes, ya que la mayoría están en ejercicio, lo que nos lleva a pensar que podrían 
haber respondido pensando más en su experiencia profesional que en lo que les estén aportando los estudios. Por ello y como propuesta de futura línea de investigación, sería interesante indagar sobre las aportaciones entorno a la formación de postgrado cursada y su efectividad a la hora de que los profesores cuenten con las herramientas necesarias para incluir estrategias integrales apoyadas en el uso de las TIC, ya sea gamificación o cualquier otra opción didáctica.

Para finalizar y en línea con lo expuesto, se detectan dos limitaciones que es preciso tener en consideración de cara a futuras líneas de investigación como la descrita con anterioridad. La primera se debe a la necesidad conocer e indagar sobre la influencia de la edad de los participantes en los resultados. Explorar las diferencias que pudieran darse por generaciones, uso y acceso de la tecnología podría ofrecer información valiosa sobre retos concretos, potencialidades y dificultades asociadas a perfiles más específicos. En cuanto a la segunda cuestión resulta pertinente identificar de manera satisfactoria el nivel educativo donde ejercen su labor en vistas a la realización de un análisis diferenciado por niveles educativos. Téngase en cuenta que, si bien esta información fue indicada en una parte importante de las reflexiones, algunos pasaron por alto este requerimiento en la actividad resultando complicado establecer resultados atendiendo a estos grupos de interés. Como propuesta a futuro, se incorporará una tabla con los datos de identificación precisos para favorecer la respuesta de estas cuestiones, invitando así a la reflexión por parte de los participantes teniendo en cuenta dichas variables.

\section{Referencias}

Álvarez, I., \& Kilbourn, B. (2005). La integración de las tecnologías de la información y la comunicación en la formación del profesorado: desafíos para enseñanza y aprendizaje constructivista. Revista Iberoamericana de Educación, 36(11), 1-17. https://doi.org/10.35362/rie36112747

Ballesta, J.(1993). Las nuevas tecnologías aplicadas a la educación, un reto para la formación inicial del profesorado. Revista Interuniversitaria de Formación del Profesorado, 16, 125-132.

Cabero, J., \& Marín, V. (2014). Miradas sobre la formación del profesorado en Tecnologías de Información y Comunicación (TIC). Enl@ce: Revista Venezolana de Información, Tecnología y Conocimiento, 11(2), 11-24.

Cabero, J., \& Valencia, R. (2021). Y el COVID-19 transformó al sistema educativo: reflexiones y experiencias por aprender International Journal of Educational Research and Innovation (IJERI), 15, 218-228. https://doi.org/10.46661/ijeri.5246

Carpena, N., Cataldi, M. \& Muñiz, G. (2012). En busca de nuevas metodologías y herramientas aplicables a la educación. Repensando nuestro rol docente en las aulas. Novos sistemas de produçao, 633-635.

Cortizo, J.C., Carrero, F., Monsalve, B., Velasco, A., Díaz, L.I., \& Pérez, J. (2011). Gamificación y docencia: lo que la universidad tiene que aprender de los videojuegos. En VIII Jornadas Internacionales de Innovación Universitaria. Retos y oportunidades del desarrollo de los nuevos títulos en educación superior. Universidad Europea de Madrid.

Cruz, J.G., \& Otero, N. (2017). Aprender jugando experiencia en el aula. El bucio: Revista digital del CEP Tenerife Sur, 32-35. 
De la Cruz, C. \& Palaoag, T. D. (2019). An empirical study of gamified learning application engagement to exceptional learners. In Proceedings of the 8th International Conference on Informatics, Environment, Energy and Applications (IEEA '19). Association for Computing Machinery, 263-267. https://doi.org/10.1145/3323716.3323762

Ferrer, S.C., Fernández, M., Polanco, N.D., Montero, M.E., \& Caridad, E.E. (2018). La gamificación como herramienta en el trabajo docente del orientador: innovación en asesoramiento vocacional desde la neurodidáctica. RIED Revista Iberoamericana de Educación, 78(1), 165-182.

Fuentes, M., \& González, J. (2017). Necesidades formativas del profesorado de Secundaria para la implementación de experiencias gamificadas en STEM. RED, Revista de Educación a Distancia, 17(54), 1-25.

Gabarda, V., Marín, D. y Romero, Ma M. (2020). La competencia digital en la formación inicial docente. Percepción de los estudiantes de Magisterio de la Universidad de Valencia. ENSAYOS. Revista de la Facultad de Educación de Albacete, 35(2), 1-16.

Gabarda, V., Rodríguez, A. \& Moreno, Mª D. (2017). La competencia digital en estudiantes de magisterio. Analisis competencial y percepción personal del futuro maestro. Educativo Siglo XXI, 35(2), 253-274. https://doi.org/10.6018/j/298601

Gairín, J. \& Castro, D. (2016). El impacto de las TIC en el aula desde la perspectiva del profesorado. Universitat Autònoma de Barcelona.

García-Vandewalle, J.M. (2017). Experiencia de gamificación de contenidos matemáticos mediante juegos de cartas evitando la brecha digital y fomentando la socialización. En Redined (Ed.), Conference Proceedings EDUNOVATIC 2017 (pp. 765-770). Adaya Press.

Garmen, P., Rodríguez, C., García-Redondo, P., \& San-Pedro-Veledo, J.C. (2019). Inteligencias múltiples y videojuegos: Evaluación e intervención con software TOI. Comunicar, 7(58), 95-104. https://doi.org/10.3916/C58-2019-09.

Gil, J. (2019). Interconectados apostando por la construcción colectiva del conocimiento. Aprendizaje móvil en Educación Infantil y Primaria. Píxel-BIT Revista de Medios y Educación, (54), 185-203. https://doi.org/10.12795/pixelbit.2019.i54.10.

Hernández, D., Ramírez-Martinell, A. \& Cassany, D. (2014). Categorizando los usuarios de sistemas digitales. Pixel-Bit. Revista de Medios y Educación, 44, 113-126. http://dx.doi.org/10.12795/pixelbit.2014.i44.08

Hernández, I. (2018). El Ministerio de Robin Hood: una experiencia de gamificación. Números Revista de Didáctica de la Matemática, 98, 153-162.

Instituto Nacional de Tecnologías Educativas y de Formación del Profesorado (2017). Marco común de Competencia Digital Docente. Ministerio de Educación.

Johnson, L., Adams, S., Estrada, V., \& Freeman, A. (2014). NMC Horizon Report: 2014 K-12 Edition. The New Media Consortium.

Kapp, K. (2012). The Gamification of learning and instruction. Pfeiffer.

Lee, J., \& Hammer, J. (2011). Gamification in education: What, how, why bother? Academic ExchangeQuarterly, 15(2), 146. 
Marín, D., Vidal, I., Peirats, J., \& San Martín, A. (2019). Competencia digital transversal en la formación del profesorado, análisis de una experiencia. Innoeduca. international Journal of technology and educational innovation, 5(1), 4-12. https://doi.org/10.24310/innoeduca.2019.v5i1.4890

Marín, V. (2015). La Gamificación educativa. Una alternativa para la enseñanza creativa. Digital Education Review, (27), 5-8.

Maté, D. (2021). Game studies: apuntes para un estado de la cuestión. En O. Echevarría (Dir.), Cuaderno 98 (pp. 19-35). Cuadernos del Centro de Estudios en Diseño y Comunicación, Universidad de Palermo.

McGonigal, J. (2011). Reality is broken: Why Games make us better and how they can change the world. Penguin.

Obando-Bastidas, J.A., Pabón, J.E., Montenegro, G., \& Castellanos, M.T. (2018). Simulacro: App, mobile application that use the innovator concept «gamifications in education» that generates massive participation in students of Colombia. Espacios, 39(53), 8-20.

OCDE (2003). La definición y selección de competencias. Resumen ejecutivo. Recuperado de http://comclave.educarex.es/pluginfile.php/130/mod_resource/content/3/DESECO.p $\mathrm{df}$

Peirats, J., Marín, D., \& Vidal, I. (2019). Bibliometría aplicada a la gamificación como estrategia digital de aprendizaje. Revista de Educación a Distancia, (60), 1-26.

Peirats, J., Muñoz, J. L. \& San Martín, A. (2015). Los imponderables de la tecnología educativa en la formación del profesorado. Revista Latinoamericana de Tecnología Educativa, 14(3), 11-22. https://doi.org/10.17398/1695-288X.14.3.11

Peirats, J., Rodríguez, J., \& San Martín, A. (2019). Controversias del liderazgo escolar en la implantación de materiales digitales. Campus Virtuales, 8(2), 19-34.

Pérez-López, I.J. (2018). La docencia es un juego donde gana el que más disfruta. Habilidad Motriz, 50, 2-3.

Prensky, M. (2001). Digital Natives, Digital Inmigrants. On the Horizon, 9(5), 1-6. https://doi.org/10.1108/10748120110424816

Prieto, E. (2018). Gamificación, motivación y aprendizaje en Educación Primaria (Tesis de maestría). Universidad Nacional de Educación a Distancia, España.

Putz, L. M., Hofbauer, F., \& Treiblmaier, H. (2020). Can gamification help to improve education? Findings from a longitudinal study. Computers in Human Behavior, 110, 106392. https://doi.org/10.1016/j.chb.2020.106392

Quintero, L.E., Jiménez, F., \& Area, M. (2018). Más allá del libro de texto. La gamificación mediada con TIC como alternativa de innovación en Educación Física. Retos, (34), 343-348.

Rodríguez, F., \& Santiago, R. (2015). Gamificación. Cómo motivar a tu alumnado y mejorar el clima en el aula. Editorial Océano.

Roig-Vila, R., Urrea-Solano, M., \& Merma-Molina, G. (2021). La comunicación en el aula universitaria en el contexto del COVID-19 a partir de la videoconferencia con Google Meet. RIED. Revista Iberoamericana de Educación a Distancia, 24(1), 197-220. http://dx.doi.org/10.5944/ried.24.1.27519 
Romero, M M., Peirats, J., San Martín, A. \& Gallardo, I. Mª (2014). Percepciones en torno al coordinador TIC en los Centros Educativos Inteligentes. Un estudio de caso. Educar, 50(1), 167-184.

Salvador-García, C. (2021). Gamificando en tiempos de coronavirus: el estudio de un caso. RED Revista de Educación a Distancia, 21(65), 1-20. https://doi.org/10.6018/red.439981

Sánchez, F. J. (2015). Gamificación. Education in the Knowledge Society EKS, 16(2), 13-15.

Silva, R., Rodrigues, R., \& Leal, C. (2020). Gamification in management education-A literature mapping. Education and Information Technologies, 25(3), 1803-1835.

Subinas, A., \& Berciano, A. (2019). La motivación en el aula de matemáticas: ejemplo de Yincana $5^{\circ}$ de Educación Primaria. Números, Revista de Didáctica de las Matemáticas, $101,45-58$.

Vázquez-Ramos, F.J. (2021). Una propuesta para gamificar paso a paso sin olvidar el currículum: modelo Edu-Game. Retos, (39), 811-819. https://doi.org/10.47197/retos.voi39.76808

Vergara, D., Mezquita, J.M., \& Gómez, A.I. (2019). Metodología innovadora basada en la gamificación educativa: evaluación tipo test con la herramienta Quizizz. Profesorado, Revista de Currículum y Formación del Profesorado, 23(3), 363-387. https://doi.org/10.30827/profesorado.v23i3.11232

Villena, R., Cózar, R., \& González-Calero, J.A. (2018). Gamificación en grupos interactivos para enseñar y aprender historia. Una experiencia en educación primaria. En J. Monteagudo-Fernández, A. Escribano-Miralles, \& C.J. Gómez-Carrasco (Eds.), Educación histórica y competencias transversales: narrativas, tic y competencia lingüística (pp. 275-286). Universidad de Murcia, Servicio de Publicaciones. 